\title{
Variables psicológicas y educativas en el
}

\section{abandono universitario}

\section{José Tomás Bethencourt Benítez ${ }^{1}$, Lidia Cabrera Pérez ${ }^{2}$, Juan Andrés Hernández Cabrera ${ }^{3}$, Pedro Álvarez Pérez ${ }^{4}$, Miriam González Afonso ${ }^{5}$}

\footnotetext{
${ }^{1}$ Departamento de Psicología Evolutiva y de la Educación, Universidad de La Laguna, Tenerife 2, 4 y 5 Departamento de Didáctica e Investigación Educativa, Universidad de La Laguna, Tenerife ${ }^{3}$ Departamento de Psicobiología y Metodología de las Ciencias del Comportamiento, Universidad de La Laguna, Tenerife
} Islas Canarias, España jbethen@ull.es

Correspondencia: Dr. José Tomás Bethencourt Benítez. Universidad de La Laguna, Dpto de Psicología Evolutiva y de la Educación. Facultad de Psicología, Campus de Guajara. Tenerife. 38071. Canarias. E-mail: jbethen@ull.es

(C) Education \& Psychology $\mathrm{I}+\mathrm{D}+\mathrm{i}$ and Editorial EOS (Spain) 


\section{Resumen}

Introducción. El propósito del presente trabajo es demostrar que en las percepciones del estudiantado universitario, las variables del alumno son vistas como más relevantes que las variables del contexto en el abandono de sus estudios universitarios.

Método. La metodología utilizada fue transversal o de corte, de tipo retrospectivo. Para ello fueron entrevistados telefónicamente 558 estudiantes mediante un cuestionario que incluía ítems sobre estrategias y actividades de estudio, características psicológicas, características de la titulación y características del profesorado.

Resultados. Los datos recogidos fueron sometidos a análisis multivariado de varianza, análisis discriminante y análisis de regresión logística. Los resultados confirman la superioridad de las variables del alumno frente a las variables del contexto en el abandono de los estudios universitarios.

Discusión. La persistencia del estudiantado universitario ha demostrado ser muy relevante en la evitación del abandono. Estos resultados son interpretados a la luz de los constructos que están siendo investigados por el movimiento mundial de la psicología positiva, como el optimismo, la esperanza, la felicidad o la resiliencia.

Palabras Clave: Abandono universitario, educación superior, características psicológicas, características educativas.

Recibido: 07/11/07 Aceptación provisional: 08/12/07 Aceptación definitiva: 13/03/08 


\begin{abstract}
Introduction, The purpose of this research is to demonstrate that on the perceptions of university students, the student variables are seen as most important than the context variables to dropout their university studies.
\end{abstract}

Method. The used methodology was cross-sectional or of cut, of retrospective type. 558 undergraduates were interviewed by telephone on study strategies and activities, psychological characteristics, characteristics of course studies and characteristics of the educators.

Results. A multivariate analysis approach was used to confirm our hypothesis. The results confirm the superiority of the student variables front the context variables to dropout the university studies.

Discusión. The students' persistence have demonstrated to be very relevant in the avoidance of the dropout. These results are interpreted in the light of the constructs that are being investigated by the world movement of the positive psychology, as the optimism, the hope, the happiness or the resilience.

Keywords: University dropout, Higher education, Psychological characteristics, Educational characteristics.

Received: 07/11/07 Initial Acceptance: 08/12/07 Definitive Acceptance: 13/03/08 


\section{Introducción}

La deserción por parte del alumnado universitario de sus estudios es uno de los múltiples indicadores de la calidad del sistema educativo, que pone de relieve la existencia de serios fallos en los procesos de incorporación, adaptación y promoción del estudiantado. Este fenómeno problemático del sistema de enseñanza superior parece ser común a muchas universidades del planeta, tal como queda patente en el informe de la UNESCO (2006), quien a través de la Asociación Internacional de Universidades (IAU) constata esa realidad en 180 países.

En el caso concreto del Estado Español, la prevalencia actual del problema del abandono universitario ha sido analizada profusamente por Cabrera, Bethencourt, Álvarez y González (2006a) quienes señalan una tasa general promedio de abandono del $26 \%$, siendo variable dicho valor en función del área de conocimiento al que pertenezcan las titulaciones consideradas, de modo que las ramas del conocimiento donde se registran mayores índices de deserción son las humanidades, seguidas de las enseñanzas técnicas y ciencias experimentales, las ciencias sociales con tasas algo inferiores y las ciencias de la salud con las tasas más bajas.

En la literatura científica internacional, Longden (2002) ha observado la gran diversidad de términos utilizados para referirse a la problemática del abandono de los estudios universitarios. Concretamente este autor ha encontrado las siguientes denominaciones: Attrition, Drop out, Failure, Interruption of study, Leaving early, Non-achievement, Non-completion, Non-persistence, Stop out, Survival, Suspension, Wastage, y Withdrawal. Estas denominaciones se refieren tanto a abandonos voluntarios como involuntarios motivados por diversas causas.

La investigación previa llevada a cabo sobre el abandono universitario se ha centrado especialmente en la identificación de las causas y las posibles soluciones a tal problema. Las causas han sido consideradas desde cuatro modelos teóricos: (a) el modelo de adaptación sostiene que el abandono se produce debido a una deficiente adaptación e integración del estudiante al ambiente académico y social de la enseñanza universitaria (Landry, 2003; Metz, 2002; Nora, 2002; Pascarella y Terenzini, 1991; Tinto, 1975, 1989, 1993); (b) el modelo estructural que entiende la deserción como el resultado de las contradicciones de los diferentes 
subsistemas (político, económico y social) que integran al sistema social en su conjunto. Los estudios que se han emprendido desde este modelo mantienen un posicionamiento crítico respecto al papel reproductor de las condiciones sociales que lleva a cabo la enseñanza universitaria (Luján y Resendiz, 1981); (c) el modelo economicista postula que el abandono se debe a la elección por parte del estudiante de una forma alternativa de invertir tiempo, energía y recursos que pueda producirle en el futuro beneficios mayores respecto a los costos de permanencia en la universidad (Albert y Toharia, 2000; Thurow, 1973); (d) el modelo psicoeducativo encuentra explicaciones al abandono universitario en las características psicológicas y educativas del estudiantado (Cabrera, Bethencourt, González y Álvarez, 2006b; González, Álvarez, Cabrera y Bethencourt, 2007; Kirton, 2000; Ryan y Glenn, 2003; Wasserman, 2001; Yip y Chung, 2005).

De los resultados obtenidos en la revisión de las investigaciones previas, se destaca que las causas específicas del abandono que han sido identificadas son abundantes y diversas, lo que demuestra la naturaleza multifactorial de dicho fenómeno. En general, las variables causales pueden ser agrupadas en los siguientes tipos: (a) Psicológicas; (b) Educativas; (c) Institucionales u Organizacionales; (d) Familiares; y (e) Comunitarias o Sociales. Esta clasificación de las causas permite reducirlas a dos grandes grupos de variables, las del alumno, más propias de las características personales del estudiantado universitario, y las del contexto, concernientes con las peculiaridades del ambiente académico y social.

Más concretamente y para una mayor claridad al lector, se debe señalar que las variables específicas asociadas al abandono de los estudios universitarios encontradas en investigaciones previas son: la baja autoeficacia (Ours y Ridder, 2003; Peterson, 2002; Wasserman, 2001); la baja motivación (Landry, 2003); la insatisfacción (Mashburn, 2001); las bajas expectativas sobre los resultados académicos (Landry, 2003); dificultades en la transición de etapa educativa (Kirton, 2000); la escasa camaradería estudiantil (Rullman, 2002); la percepción de la baja utilidad del grado (Peterson, 2002); creencias negativas sobre sí mismo (Gloria y Kurpius, 2001); experiencias estudiantiles negativas (Yorke, 2000); ausencia de apoyo social (Gloria y Kurpius, 2001); mal ambiente universitario (Forbes y Wickens, 2005); dificultades o limitaciones económicas y financieras (Braunstein, McGrath y Pescatrice, 2001; Cofer y Somers, 2001; Ishitani y DesJardins, 2003); aprendizaje no activo (Braxton, Milen y Sullivan, 2000); inadaptación social (Braxton y McClendon, 2002); deficientes condiciones 
organizacionales (Berger, 2002; Braxton y Mundy, 2002; Kuh, 2002; Montmarquette, Mahseredjian y Houle, 2001; Thomas, 2002) y bajo apoyo familiar y comunitario (Nora, 2002).

Tal como se ha encontrado en la revisión de la literatura científica, las investigaciones previas se centran en demostrar la influencia de las variables, bien del sujeto o bien del contexto separadamente, pero no abordan conjuntamente el peso diferencial de ambos tipos de variables en el abandono universitario. La comparación directa y sistemática de la influencia diferencial de ambos grupos de variables (sujeto versus contexto) en el abandono universitario no ha sido considerada en la investigación previa.

El objetivo pues del presente trabajo es demostrar que en las percepciones del estudiantado universitario, las variables del alumno son vistas como más relevantes que las variables del contexto en el abandono de sus estudios universitarios. Se basa dicha hipótesis en los hallazgos de la investigación de Taylor y Bedford (2004), quienes descubrieron que en las percepciones del profesorado universitario sobre las causas de la no terminación de los estudios, los factores relacionados con las características del estudiantado son más importantes que los factores concernientes con la práctica educativa o el desarrollo curricular.

\section{Método}

\section{Participantes}

La muestra estuvo constituida por 558 estudiantes de la Universidad de La Laguna (Canarias, España), con una edad media de 26 años, de los cuales el 61,8\% eran mujeres y el $38,2 \%$ hombres. Para el 78,3\% de los encuestados la titulación universitaria en la que se habían matriculado era la primera. En el momento de recoger los datos el 52\% había prolongado, es decir, seguía cursando los estudios o los había terminado empleando más tiempo del prescrito; el 28\% abandonó los estudios y sólo el 20\% había terminado en el tiempo prescrito.

Respecto al proceso de muestreo de los sujetos seleccionados, la muestra definitiva de 558 estudiantes universitarios fue obtenida, por un lado, de la promoción de alumnos que en el curso académico 1998/99 se habían matriculado en el primer año de carreras de ciclo largo (21 títulos), y por otro lado, de la promoción de alumnos que en el curso académico 1999/00 se habían matriculado en el primer año de carreras de ciclo corto (18 títulos). Esas dos cohor- 
tes estaban compuesta por un total de 4.634 estudiantes. A partir de ellas se hizo un muestreo aleatorio estratificado a un nivel de confianza del $99,7 \%$ y un error del $4 \%$.

Instrumento.

Para la recogida de información se utilizó el Cuestionario de Abandono de Estudios Universitarios, CADEU (Cabrera, Bethencourt, Álvarez y González, 2007), que mide las percepciones del alumnado universitario sobre sus estrategias y actividades de estudio, sus características psicológicas, las características de la titulación cursada y las características del profesorado.

El primer apartado del cuestionario sobre las estrategias y actividades de estudio del alumnado estuvo formado por 17 ítems, presentando una consistencia interna medida con el $\alpha$ de Crombach de 0.75. El apartado de características psicológicas estuvo formado por 7 ítems, presentando una consistencia interna de $\alpha=0.78$. El apartado de características de la titulación estuvo formado por 10 ítems, dando lugar a una consistencia interna de $\alpha=0.70$. Finalmente, el apartado de características del profesorado estuvo formado por 12 ítems, presentando una consistencia interna de $\alpha=0.87$.

Todos los ítems de los cuatro apartados del cuestionario ya señalados fueron presentados con una escala de diez valores de respuesta, donde el 0 equivalía a nunca o muy bajo y el valor 9 a siempre o muy alto.

\section{Diseño}

Se decidió realizar esta investigación con una muestra de dos cohortes de estudiantes que habían iniciado estudios en la Universidad de la Laguna, cada una de ellas en un mismo curso académico, dejando transcurrir para cada cohorte dos años más del tiempo prescrito para la culminación de los estudios, para de ese modo poder constatar las tres situaciones académicas posibles, a saber, terminación en el tiempo prescrito, prolongación del tiempo de los estudios y abandono de los mismos. Para ello se utilizó una metodología transversal o de corte, de tipo retrospectivo, que permitió hacer el seguimiento de la relación entre las variables predictivas (factores determinantes del abandono de los estudios) y la criterio (el abandono de los estudios), reconstruyendo el pasado a partir de los datos recogidos en el presente. Las variables objeto de estudio se revisaron, por tanto, de forma simultánea, instando a los encuestados a recordar sobre dichas circunstancias en el desarrollo de la carrera. La presencia 
de una covariación sistemática entre los dos tipos de variables constituyó la clave que nos permitió determinar las posibles causas explicativas.

A partir del grupo poblacional elegido se realizó un extracto muestral de las dos cohortes temporales seleccionadas. Las variables objeto de investigación, unas como predictivas y otras como criterio, fueron las siguientes:

- Variable criterio, situación académica, con tres niveles: terminación, prolongación y abandono de estudios.

- Variables predictivas. Se presentan todas como variables cuantitativas, en una escala valorativa de diez niveles (desde muy baja a muy alta). Estas variables se agrupan en torno a cuatro apartados: estrategias y actividades de estudio del alumnado; características psicológicas del alumnado; características de la titulación; características del profesorado. Debe precisarse que los dos primeros apartados de variables fueron considerados como variables del alumno y los dos restantes como variables del contexto. En la sección de resultados de este trabajo pueden observarse detalladamente la totalidad de las variables predictivas contempladas.

\section{Procedimiento}

En cuanto al procedimiento de administración del cuestionario CADEU, éste fue administrado telefónicamente durante 20 minutos aproximadamente, por tres encuestadoras debidamente entrenadas, a lo largo de los meses de Mayo, Junio y Julio del año 2004. La fecha en la que fueron recogidos los datos permitió dejar transcurrir seis cursos académicos en el caso del estudiantado de carreras de ciclo largo (4 años) y cinco cursos académicos en el caso del estudiantado de carreras de ciclo corto (3 años), esos dos años de más del tiempo prescrito para cada tipo de carrera permitiría observar las diversas situaciones académicas (terminación, prolongación o abandono), en las que se encontraría el estudiantado seleccionado.

\section{Análisis estadísticos}

Para el análisis de los datos se utilizó el paquete estadístico SPSS versión 14.0 para Windows. Primeramente se llevó a cabo un análisis multivariado de varianza, posteriormente se realizó un análisis discriminante, para luego concluir con un análisis de regresión logística. 


\section{Resultados}

Para comprobar si había diferencias significativas entre el alumnado que abandona, prolonga y termina, se realizó un análisis multivariado de la varianza (Manova) con cada uno de los cuatro conjuntos de variables estudiadas: Estrategias y actividades de estudio, Características psicológicas del alumnado, Características de la titulación y Características del profesorado. El resultado de dicho Manova llevó a desechar al grupo que prolonga por ser intermedio entre los otros dos, obtándose por tanto a considerar sólo los grupos extremos de los que terminan y abandonan. Dado que el resultado del Manova para tales grupos extremos fue significativo, se realizó un análisis discriminante con la intención de determinar el papel desempeñado por cada variable dentro de la función, y valorar el potencial de sensibilidad y precisión de la combinación lineal para averiguar a qué perfil se aproxima el alumnado (al del que abandona o al del que termina). Posteriormente, se hizo un análisis de regresión logística con el objetivo de modelar el riesgo de abandonar los estudios a partir de saltos percentílicos en las variables covariables del modelo logístico, utilizando el último cuartil (> P75) como categoría de referencia. Se presenta a continuación el comportamiento de cada uno de los constructos estudiados.

\section{Estrategias y Actividades de Estudio}

Esta escala formada por 17 ítems presenta una consistencia interna medida $\alpha=0.75$, y los coeficientes de estructura de cada ítem garantizaron la correlación de cada uno de ellos con la función. El análisis multivariado de la varianza realizado para este grupo de variables produjo una lambda de Wilks de $0.58, F(17,271)=10,37(\mathrm{p}<0.001)$ con un tamaño del efecto global muy grande $\left(\eta^{2}=0.413\right)$, lo que indica que la proporción de varianza explicada es superior al 40\%. Como puede verse en la Tabla 1, las variables que mejor quedan explicadas por dicha función son las que presentan un tamaño de efecto univariado y un coeficiente de estructura mayor: "Realizar actividades de formación complementaria", "Plantear dudas al profesor", "Repasar los temas estudiados", "Llevar los contenidos de clase al día” y "Asistir regularmente a clase y a tutorías". Ante estos resultados se puede estimar que los estudiantes que más realizan estas actividades tienen más posibilidades de ser persistentes con los estudios y terminar la carrera. 
Tabla 1. Estrategias y actividades de estudio

\begin{tabular}{|c|c|c|c|c|}
\hline & $\mathrm{F}(1,267)$ & $\eta^{2}$ & $\mathrm{CE}$ & $\mathrm{CT}$ \\
\hline Asistir a clases & $26.41 * * *$ & .090 & 0.41 & .228 \\
\hline Plantear dudas al profesorado durante sus explicaciones & $46.17 * * *$ & .147 & 0.36 & .188 \\
\hline Tener en cuenta recomendaciones del profesorado al estudiar & $11.69 * * *$ & .041 & 0.29 & -.146 \\
\hline Realizar lectura previa al comenzar a estudiar un tema & $7.42 * *$ & .027 & 0.15 & -.038 \\
\hline Realizar anotaciones en el texto cuando se estudia & $13.03 * * *$ & .046 & 0.17 & .044 \\
\hline Subrayar el texto para resaltar ideas más importantes & $12.15^{* * *}$ & .043 & 0.13 & .107 \\
\hline Hacer esquemas o resúmenes de los temas & $8.83 * *$ & .032 & 0.17 & -.007 \\
\hline Repasar los temas estudiados & $31.33 * * *$ & .105 & 0.26 & .155 \\
\hline Utilizar otras técnicas de estudio (Síntesis, Cuadros, Diagramas) & $6.00 * *$ & .022 & 0.30 & -.181 \\
\hline Estudiar de memoria sin comprender & 3.02 & .011 & -0.03 & -.100 \\
\hline Utilizar otros materiales complementarios para estudiar & 2.01 & .007 & 0.22 & -.161 \\
\hline Consultar Internet para buscar información & $15.86^{* * *}$ & .056 & 0.18 & .108 \\
\hline Llevar los contenidos de las asignaturas al día & $27.23 * * *$ & .092 & 0.30 & .050 \\
\hline Asistir a tutorías & $25.52 * * *$ & .087 & 0.23 & .133 \\
\hline Realizar actividades de formación complementaria & $121.91 * * *$ & .313 & 0.88 & .763 \\
\hline Analizar las causas de resultados no esperados & $11.72 * * *$ & .042 & 0.27 & .038 \\
\hline Dejar de estudiar por no comprender la materia & $18.78^{* * *}$ & .065 & 0.35 & .241 \\
\hline
\end{tabular}

$*(\mathrm{p}<0.05), * *(\mathrm{p}<0.01), * * *(\mathrm{p}<0.001)$

En lo que a los coeficientes de estructura se refiere, puede verse que son esas mismas variables las que más aportan a la función discriminante con la salvedad de la variable "Llevar los contenidos al día" que presenta un valor muy bajo, lo cual la señala como redundante. En cuanto a los centroides o medias de la función discriminante por grupo, el grupo que "Termina" presenta un valor significativamente mayor (0.99), que el grupo que "Abandona" (-0.70).

Para el análisis de regresión logística que se realizó a continuación, se seleccionó como variables covariables (predictoras en la función logística) las dos más importantes: "Realizar formación complementaria" y "Plantear dudas al profesorado". La función logística resultante fue significativa (ver Tabla 2). Ambas variables se muestran, por tanto, predictoras del riesgo de abandono, dado que presentan una exponencial del parámetro de regresión inferior a $1(\operatorname{Exp}(B)=0.36$ y 0,62 respectivamente $)$. 
Tabla 2. Regresión logística en las variables de estrategias y actividades de estudio

\begin{tabular}{|c|c|c|c|c|c|c|}
\hline \multicolumn{7}{|c|}{$\chi^{2}(6)=115.7(p<0.001)$} \\
\hline & $\mathrm{B}$ & E.T. & Wald & $\mathrm{gl}$ & Sig. & $\operatorname{Exp}(\mathrm{B})$ \\
\hline Plantear dudas & & & 12.705 & 3 & .005 & .365 \\
\hline $\mathrm{PD}(1)$ & 1.553 & .471 & 10.848 & 1 & .001 & 4.725 \\
\hline $\mathrm{PD}(2)$ & .778 & .375 & 4.296 & 1 & .038 & 2.178 \\
\hline $\mathrm{PD}(3)$ & .075 & .519 & .021 & 1 & .886 & 1.078 \\
\hline $\begin{array}{c}\text { Formación } \\
\text { complementaria }\end{array}$ & & & 39.868 & 3 & .000 & .619 \\
\hline FC (1) & 3.640 & .590 & 37.992 & 1 & .000 & 38.074 \\
\hline $\mathrm{FC}(2)$ & 1.205 & .396 & 9.259 & 1 & .002 & 3.336 \\
\hline $\mathrm{FC}(3)$ & .662 & .412 & 2.583 & 1 & .108 & 1.939 \\
\hline Constante & -1.47 & .340 & 18.888 & 1 & .000 & .229 \\
\hline
\end{tabular}

\section{Características psicológicas del alumnado}

La segunda escala, formada por 7 ítems, presenta una consistencia interna de $\alpha=0.78$ $\mathrm{y}$, como en el caso anterior, muestra una función significativa con una lambda de Wilks de 0.41 y un estadístico $\mathrm{F}(7,261)=52.3(\mathrm{p}<0.001)$ con un gran tamaño del efecto de 0.58 $\left(\eta^{2}=0.58\right)$. La media de la función discriminante (centroide) en el grupo que termina es significativamente mayor (1.39) que en el grupo que abandona (-0.99).

Tabla 3. Características psicológicas del alumnado

\begin{tabular}{|l|l|l|l|r|}
\hline & $\mathrm{F}(1,267)$ & $\eta^{2}$ & $\mathrm{CE}$ & $\mathrm{CT}$ \\
\hline Satisfacción con la titulación cursada & $149.4 * * *$ & .358 & 0.63 & .398 \\
\hline Conocimientos previos sobre contenidos de la titulación & .02 & .000 & 0.00 & -.116 \\
\hline Motivación hacia la titulación cursada & $75.5 * * *$ & .220 & 0.45 & -.076 \\
\hline Ajuste entre capacidades y exigencias de titulación & $77.3 * * *$ & .224 & 0.45 & .316 \\
\hline Persistencia para acabar la titulación a pesar de los obstáculos & $293.7 * * *$ & .523 & 0.88 & .778 \\
\hline Capacidad para demorar la recompensa & $85.6 * * *$ & .242 & 0.48 & -.096 \\
\hline Coincidencia entre la idea previa sobre la titulación y la realidad & $18.5 * * *$ & .065 & 0.22 & -.014 \\
\hline$*(\mathrm{p}<0.05), * *(\mathrm{p}<0.01), * * *(\mathrm{p}<0.001)$ & & &
\end{tabular}

La varianza es altamente explicada (un 58\%) por cinco variables, que también saturan alto en la función discriminante: "Persistencia para acabar la carrera a pesar de los obstáculos", que destaca entre las demás con un efecto muy grande, "Satisfacción con la titulación cursada", "Capacidad para demorar las recompensas", "Motivación hacia la titulación" y “Ajuste entre capacidades y exigencias de la titulación". Los coeficientes típicos de las variables "Motivación hacia la titulación cursada" y "Capacidad para demorar la recompensa" son muy bajos, lo que demuestra el carácter redundante de esas variables en la función discriminante. 
Con las cuatro variables que más saturaban en la función multivariada, se realizó la transformación en cuatro cuartiles. Dichas variables fueron analizadas mediante el procedimiento test de Wald que condujeron hacia una función significativa con sólo dos variables: "Satisfacción con la titulación" y "Persistencia para acabar". En la Tabla 4 se muestra el efecto protector que ambas variables tienen sobre el riesgo de abandonar $(\operatorname{Exp}(B)<1)$.

Tabla 4. Regresión logística en las variables de características psicológicas del alumnado

\begin{tabular}{|c|c|c|c|c|c|c|}
\hline$\chi^{2}(6)=226.3(\mathrm{p}<0.001)$ & & & & & \\
\hline & B & E.T. & Wald & Gl & Sig. & Exp(B) \\
\hline Satisfacción & & & 16.87 & 3.00 & 0.000 & 0.485 \\
\hline ST(1) & 2.97 & 0.78 & 14.70 & 1.00 & 0.000 & 19.59 \\
\hline ST(2) & 0.52 & 0.53 & 0.98 & 1.00 & 0.320 & 1.68 \\
\hline ST(3) & -0.27 & 0.64 & 0.17 & 1.00 & 0.680 & 0.76 \\
\hline Persistencia & & & 61.82 & 3.00 & 0.000 & 0.160 \\
\hline PAT(1) & 5.27 & 0.76 & 48.25 & 1.00 & 0.000 & 193.85 \\
\hline PAT(2) & 3.00 & 0.63 & 22.85 & 1.00 & 0.000 & 20.15 \\
\hline PAT(3) & 0.78 & 0.74 & 1.12 & 1.00 & 0.290 & 2.19 \\
\hline Constante & -2.94 & 0.57 & 26.34 & 1.00 & 0.000 & 0.05 \\
\hline Categoría de referencia último cuartil & \multicolumn{7}{l}{} \\
\hline
\end{tabular}

\section{Características de la titulación}

Los 10 ítems de esta escala dieron lugar a una consistencia interna de $\alpha=0.70$. En este grupo de variables, a pesar de que la diferencia entre los que abandonan y terminan es significativa, $(\Lambda=0.78, F(10,250)=6.75(p<0.001))$, el efecto global es de $0,213\left(\eta^{2}=0.213\right)$, muy inferior a los dos constructos anteriores (ver Tabla 5). Sin embargo, los datos muestran como el alumnado que abandona percibe la carrera como más exigente, dice que recibe menos formación práctica, con contenidos menos útiles, que no tuvieron la suficiente orientación e información durante la carrera y opinan que la calidad de las relaciones con los compañeros no es tan buena. El resto de los análisis también nos indican la escasa bondad del modelo para predecir o clasificar al alumnado en relación al abandono o finalización de estudios.

Tabla 5. Características de la titulación

\begin{tabular}{|l|l|c|c|c|}
\hline & \multicolumn{1}{|c|}{$\mathrm{F}(1,259)$} & \multicolumn{1}{|c|}{$\eta^{2}$} & $\mathrm{CE}$ & $\mathrm{CT}$ \\
\hline Coherencia entre los contenidos de la titulación & 2.6 & .010 & -.194 & -.072 \\
\hline Utilidad de los contenidos para el desempeño profesional & $6.3 * *$ & .023 & -.302 & -.339 \\
\hline Exigencia de la titulación & $18.7^{* * *}$ & .067 & .518 & .622 \\
\hline Masificación en las clases teóricas & $4.4^{*}$ & .016 & .251 & .237 \\
\hline Organización y funcionamiento de la titulación & .17 & .000 & -.049 & .199 \\
\hline Calidad de la relación con los compañeros & $9.5^{* *}$ & .035 & -.369 & -.213 \\
\hline Formación práctica recibida & $11.4 * * *$ & .042 & -.404 & -.429 \\
\hline Disponibilidad de Servicios de apoyo al estudio & 3.5 & .013 & .225 & .431 \\
\hline Información y orientación recibida durante la titulación & $14.6 * * *$ & .053 & -.458 & -.332 \\
\hline Salidas laborales de la titulación & 1.1 & .004 & .125 & .089 \\
\hline
\end{tabular}

$*(\mathrm{p}<0.05), * *(\mathrm{p}<0.01), * * *(\mathrm{p}<0.001)$ 


\section{Características del profesorado}

Esta última escala de 12 ítems presenta una consistencia interna de $\alpha=0.87$. El análisis multivariado de la varianza, dio lugar a una función significativa $(\Lambda=0.76, F(12,210)=5.35$ (p $<0.001)$ ) con un tamaño de efecto global de $0.23\left(\eta^{2}=0.213\right)$. En la Tabla 6 se presentan los estadísticos resultantes de los distintos análisis realizados, y en los que encontramos que el alumnado que termina los estudios siente que el profesorado es más dialogante con el alumnado, tiene más en cuenta sus opiniones y les motivaban en los estudios.

Tabla 6. Características del profesorado

\begin{tabular}{|c|c|c|c|c|}
\hline & $\mathrm{F}(1,221)$ & $\eta^{2}$ & $\mathrm{CE}$ & $\mathrm{CT}$ \\
\hline Cumplir con el horario de clase & 0.4 & 0.00 & .075 & .049 \\
\hline Cumplir con el horario de tutoría & 0.1 & 0.00 & -.028 & -.297 \\
\hline Preparar las clases & 0.9 & 0.00 & .115 & -.227 \\
\hline Explicar los contenidos con claridad & $8.0^{* *}$ & 0.03 & .346 & -.172 \\
\hline Relacionar contenidos con la práctica profesional & $10.7 * * *$ & 0.05 & .399 & .067 \\
\hline Usar diferentes formas para trabajar en el aula & $20.9 * * *$ & 0.09 & .558 & .144 \\
\hline Dialogar con el alumnado sobre la marcha de las clases & $31.2 * * *$ & 0.12 & .682 & .342 \\
\hline Tener en cuenta las opiniones del alumnado & $34.7 * * *$ & 0.14 & .719 & .385 \\
\hline Motivar al alumnado hacia la asignatura & $35.5 * * *$ & 0.14 & .727 & .366 \\
\hline Ajustar los exámenes a los contenidos impartidos & $13.4 * * *$ & 0.06 & .447 & .323 \\
\hline Dominar el contenido enseñado & 0.3 & 0.00 & .064 & .008 \\
\hline Exigencia del profesorado & 1.7 & 0.01 & -.159 & -.282 \\
\hline
\end{tabular}

La regresión logística, como en los casos anteriores, también se realizó con estas tres variables más relevantes. Los análisis resultantes (test de Wald) condujeron a desechar la variable "Tener en cuenta las opiniones del alumnado", por lo que en la Tabla 7 se presentan los valores de las variables motivación y diálogo con el alumnado sobre la marcha de las clases, ambas con un efecto significativo $(\mathrm{p}<0.001)$. 
Tabla 7. Regresión logística en las variables de características del profesorado

\begin{tabular}{|c|c|c|c|c|c|c|}
\hline \multicolumn{2}{|c|}{$\chi 2(6)=49.2(\mathrm{p}<0.001)$} & & & & & \\
\hline & $\mathrm{B}$ & E.T. & Wald & gl & Sig. & $\operatorname{Exp(B)}$ \\
\hline Motivar alumnado & & & 15.99 & 3.00 & 0.000 & 0.61 \\
\hline MA(1) & 1.73 & 0.44 & 15.81 & 1.00 & 0.000 & 5.66 \\
\hline MA(2) & 0.69 & 0.36 & 3.57 & 1.00 & 0.060 & 1.99 \\
\hline MA(3) & 0.69 & 0.39 & 3.18 & 1.00 & 0.070 & 1.99 \\
\hline Diálogo alumnado & & & 13.18 & 3.00 & 0.000 & 0.60 \\
\hline DA(1) & 1.31 & 0.49 & 7.20 & 1.00 & 0.010 & 3.71 \\
\hline DA(2) & 1.33 & 0.43 & 9.69 & 1.00 & 0.000 & 3.77 \\
\hline DA(3) & 0.48 & 0.38 & 1.58 & 1.00 & 0.210 & 1.62 \\
\hline Constante & -1.10 & 0.33 & 11.03 & 1.00 & 0.000 & 0.33 \\
\hline Categoría de referencia último cuartil & & & & \\
\hline
\end{tabular}

\section{Discusión y Conclusiones}

Los resultados obtenidos en la presente investigación permiten confirmar que las variables del alumnado están más asociadas al abandono de los estudios universitarios que las variables del contexto.

Si se ordenan las variables analizadas según el grado de asociación percibido en el abandono universitario, se tendría que el orden sería el siguiente: (1) características psicológicas; (2) estrategias y actividades de estudio; (3) características del profesorado; y (4) características de la titulación.

La presente investigación ha permitido demostrar la importancia de ciertas características psicológicas del estudiantado universitario en la terminación de sus estudios. Parece ser que "la persistencia para acabar la titulación a pesar de los obstáculos", "la motivación hacia la titulación cursada", "la capacidad de esfuerzo, en favor de logros futuros", "el ajuste entre las capacidades del alumnado y las exigencias de la titulación", y "la satisfacción con la titulación cursada" son garantías de éxito académico. Tal conjunto de rasgos personales nos coloca frente a un estudiante persistente y perseverante que es capaz de demorar las recompensas, de superar obstáculos y dificultades, de mantener claras las metas de largo plazo, de fijar firmemente el rumbo o dirección de futuro, y, en definitiva, de ser constante en el mantenimiento de los planes establecidos.

Coincidiendo con Tinto (1993), se puede afirmar que un estudiante universitario que exhiba tales características personales es más competente para realizar buenas adaptaciones sociales y académicas, al contexto universitario en el que trata de integrarse de un modo activo y constructivo. 
La alta relevancia de la persistencia demostrada en esta investigación, lleva a relacionarla con la resiliencia psicológica, apuntando a que el estudiantado universitario que termina sus estudios es más capaz de afrontar con mayor éxito las adversidades de las situaciones académicas universitarias (Lightsey, 2006). En muchas titulaciones universitarias los estudiantes se encuentran con multitud de problemas y dificultades como exceso de asignaturas, sobrecarga de contenidos de aprendizaje, limitación temporal para responder a tareas y trabajos académicos exigidos, excesiva concentración temporal de exámenes, etc. Tales dificultades se convierten en un desafío ante el cual el estudiantado debe aprender a hacer frente exitosamente, para a pesar de esas circunstancias negativas encontrar satisfacción y sentir bienestar. La resiliencia es un constructo que ha recibido muy poca atención en la investigación sobre abandono universitario y que podría arrojar buenas explicaciones sobre los mecanismos psicológicos que operan en los estudiantes persistentes.

Asimismo, se señala que para futuras investigaciones la persistencia del alumnado universitario pudiera ser estudiada en relación con los constructos que vienen siendo analizados por parte del movimiento mundial de "la psicología positiva" (Seligman y Csikszentmihalyi, 2000; Seligman, Steen, Park y Peterson 2005), de modo que, el optimismo (Reivich y Gillham, 2003), la esperanza (Snyder, 2002), o la felicidad (Csikszentmihalyi, 2003; Lyubomirsky, King y Diener 2005; Shmotkin, 2005) pudieran encontrarse de manera significativa en tales universitarios.

Finalmente, se coincide con Braxton (2002) cuando plantea, que las soluciones efectivas al fenómeno problemático del abandono universitario vendrán por la vía de la combinación de las tres dimensiones implicadas: la personal, la sociológica y la organizacional. Asimismo, la acumulación de datos y resultados en la investigación sobre el abandono universitario tendrá consecuencias prácticas de cara a su solución, permitiendo la implementación de estrategias y políticas como las recogidas por Feldman (2005) en su reciente trabajo de revisión. 


\section{Referencias}

Albert, C., y Toharia, L. (2000). El abandono o la persistencia en los estudios universitarios. Papeles de Economía Española, 86, 192-212.

Berger, J. B. (2002). Understanding the organizational nature of student persistence: Empirically-based recommendations for practice. Journal of College Student Retention: Research, Theory \& Practice, 3(1), 3-21.

Braunstein, A., McGrath, M., y Pescatrice, D. (2001). Measuring the impact of financial factors on college persistence. Journal of College Student Retention, 2(3), 191-203.

Braxton, J. M. (2002). Introduction to special issue: Using theory and research to improve college student retention. Journal of College Student Retention: Research, Theory \& Practice, 3(1), 1-2.

Braxton, J. M., y McClendon, S.A. (2002). The fostering of social integration and retention through institutional practice. Journal of College Student Retention: Research, Theory \& Practice, 3(1), 57-71.

Braxton, J. M., Milen, J. F., y Sullivan, A. S. (2000). The influence of active learning on the college student departure process: toward a revision of Tinto's theory. Journal of Higher Education, 71(5), 569-590.

Braxton, J. M., y Mundy, M. E. (2002). Powerful institutional levers to reduce college student departure. Journal of College Student Retention: Research, Theory \& Practice, 3(1), 91118.

Cabrera, L., Bethencourt, J. T., Álvarez, P., y González, M. (2006a). El problema del abandono de los estudios universitarios. Relieve. Revista Electrónica de Investigación y Evaluación Educativa, 12(2). http://www.uv.es/RELIEVE/v12n2/RELIEVEv12n2 1.htm Texto consultado en Septiembre de 2007.

Cabrera, L., Bethencourt, J. T., González, M., y Álvarez, P. (2006b). Un estudio transversal retrospectivo sobre prolongación y abandono de estudios universitarios. Relieve. Revista Electrónica de Investigación $y$ Evaluación Educativa, 12(1). http://www.uv.es/RELIEVE/v12n1/RELIEVEv12n1 1.htm. Texto consultado en Septiembre de 2007.

Cabrera, L., Bethencourth, J. T., Álvarez, P., y González, M. (2007). Cuestionario de abandono de estudios universitarios (CADEU): características y estructura. Comunicación presentada al XIII Congreso Nacional de Modelos de Investigación Educativa: Convivencia, Equidad, Calidad. San Sebastián, del 27 al 29 de Junio de 2007. 
Cofer, J., y Somers, P. (2001). What influences student persistence at two-year colleges? Community College Review, 29(3), 56-76.

Csikszentmihalyi, M. (2003). Happiness in everyday life: the uses of experience sampling. Journal of Happiness Studies, 4(2), 185-199.

Feldman, R. S. (2005). Improving the first year of college: Research and practice. Mahwah, NJ: Lawrence Erlbaum Associates.

Forbes, A., y Wickens, E. (2005). A good social life helps students to stay the course. Times Higher Education Supplement, 1676, 58-63.

Gloria, A. B., y Kurpius, S. E. R. (2001). Influences of self-beliefs, social support and comfort in the university environment on the academic nonpersistence decisions of American Indian undergraduates. Cultural Diversity \& Ethnic Minority Psychology, 7(1), 88-102.

González, M., Álvarez, P., Cabrera, L., y Bethencourt, J. T. (2007). El abandono de los estudios universitarios: factores determinantes y medidas preventivas. Revista Española de Pedagogía, LXV(236), 71-86.

Ishitani, T. T., y DesJardins, S. L. (2003). A longitudinal investigation of dropout from college in the United States. Journal of College Student Retention: Research, Theory \& Practice, 4(2), 173-201.

Kirton, M. J. (2000). Transitional factors influencing the academic persistence of firstsemester undergraduate freshmen. Dissertation Abstracts International Section A: Humanities \& Social Sciences, 61(2-A), pp. 522.

Kuh, G. D. (2002). Organizational culture and student persistence: Prospects and puzzles. Journal of College Student Retention: Research, Theory \& Practice, 3(1), 23-39.

Landry, C. C. (2003). Self-efficacy, motivation and outcome expectation correlates of college students' intention certainty. Dissertation Abstracts International Section A: Humanities \& Social Sciences, 64, 3-A, pp. 825.

Lightsey, O. R. (2006). Resilience, meaning and well-being. Counseling Psychologist, 34(1), 96-107.

Longden, B. (2002). Retention rates - renewed interest but whose interest is being served? Research Papers in Education, 17(1), 3-29.

Luján, J. R., y Resendiz, A. N. (1981). Hacia la construcción de un modelo causal en el análisis de la deserción. México D.F.: Universidad Autónoma Metropolitana Iztapalapa.

Lyubomirsky, S., King, L., y Diener, E. (2005). The benefits of frequent positive affect: Does happiness lead to success? Psychological Bulletin, 131(6), 803-855. 
Mashburn, A. J. (2001). A psychological process of college student dropout. Journal of College Student Retention, 2(3), 173-190.

Metz, G. W. (2002). Challenges and changes to Tinto's persistence theory. Paper presented at the Annual Meeting of the Mid-Western Educational Research Association. Columbus, OH, October 16-19.

Nora, A. (2002). The depiction of significant others in Tinto's "rites of passage": A reconceptualization of the influence of family and community in the persistence process. Journal of College Student Retention: Research, Theory \& Practice, 3(1), 41-56.

Ours, J. C., y Ridder, G. (2003). Fast track or failure: A study of the graduation and dropout rates of Ph D students in economics. Economics of Education Review, 22, 157-166.

Pascarella, E. T., y Terenzini, P. T. (1991). How college affects students: Findings and insights from twenty years of research. San Francisco, CA: Jossey Bass.

Peterson, S. L. (2002). Effects of career decision-making, self-efficacy and degree utility on student persistence: A path analytic study. Journal of College Student Retention: Research, Theory \& Practice, 3(3), 285-299.

Reivich, K., y Gillham, J. (2003). Learned optimism: The measurement of explanatory style. En S. J. Lopez y C. R. Snyder (Eds.) Positive psychological assessment: A handbook of models and measures (pp. 57-74). Washington, DC: American Psychological Association.

Rullman, L. J. (2002). Research review shows connection between fraternity, sorority involvement and retention. Bulletin, 70(5), 35-38.

Ryan, M. P., y Glenn, P. A. (2003). Increasing one-year retention rates by focusing on academic competence: An empirical odyssey. Journal of College Student Retention, 4(3), 297-324.

Seligman, M. E., y Csikszentmihalyi, M. (2000). Positive psychology: An introduction. American Psychologist, 55(1), 5-14.

Seligman, M. E., Steen, T. A., Park, N., y Peterson, C. (2005). Positive psychology progress: Empirical validation of interventions. American Psychologist, 60(5), 410-421.

Shmotkin, D. (2005). Happiness in the face of adversity: Reformulating the dynamic and modular bases of subjective well-being. Review of General Psychology, 9(4), 291-325.

Snyder, C. R. (2002). Hope theory: Rainbows in the mind. Psychological Inquiry, 13(4), 249275.

Taylor, J. A., y Bedford, T. (2004). Staff perceptions of factors related to non-completion in higher education. Studies in Higher Education, 29(3), 375-394. 
Thomas, L. (2002). Student retention in higher education: The role of institutional habitus. Journal of Education Policy, 17(4), 423-442.

Tinto, V. (1975). Dropout from higher education: A theoretical synthesis of recent research. Review of Educational Research, 45, 89-125.

Tinto, V. (1989). Definir la deserción: una cuestión de perspectivas. Revista de Educación Superior. México D. F., Asociación Nacional de Universidades e Instituciones de Educación Superior (ANUIES), vol. XVIII, No 3.

Tinto, V. (1993). Leaving college: Rethinking the causes and cures of student attrition. Chicago: University of Chicago Press.

Thurow, L. (1973). The political economy of income redistribution. Annals of the American Academy of Political and Social Science, 409, 146-155.

UNESCO (2006). World higher education database 2006/7. Disponible en: http://www.unesco.org/iau/directories/index.html Consultado en Septiembre de 2007.

Wasserman, K. N. (2001). Psychological and developmental differences between students who withdraw from college for personal-psychological reasons and continuing students. Dissertation Abstracts International Section A: Humanities \& Social Sciences, 62(3-A). pp. 915.

Yip, M. C. W., y Chung, O. L. L. (2005). Relationship of study strategies and academic performance in different learning phases of higher education Hong Kong. Educational Research and Evaluation, 11(1), 61-70.

Yorke, M. (2000). The quality of the student experience: What can institutions learn from data relating to non-completion? Quality in Higher Education, 6(1), 61-75. 
[Página en blanco por razones de paginación] 